Check for updates

Kent

Cite this as: BMJ 2021;374:n1914 http://dx.doi.org/10.1136/bmj.n1914 Published: 29 July 2021

\title{
Covid-19: NICE issues guidance on vaccine induced immune thrombocytopenia and thrombosis
}

Jacqui Wise

Patients who are acutely unwell with suspected vaccine induced immune thrombocytopenia and thrombosis (VITT) should be referred immediately to the emergency department, says new rapid guidance from the National Institute for Health and Care Excellence (NICE). ${ }^{1}$

However, a full blood count should be performed in primary care if the patient is not acutely unwell and if same day results can be obtained. If these blood tests show a low platelet count the patient should be referred to the emergency department on the same day.

NICE says that although VITT is extremely rare, with 14.2 cases per million doses of a covid-19 vaccine, the condition requires swift diagnosis and urgent treatment.

As of 14 July the Medicines and Healthcare Products Regulatory Agency had received "yellow card” reports of 411 cases of major thromboembolic events with concurrent thrombocytopenia after vaccination with the AstraZeneca vaccine and 15 cases after the Pfizer-BioNTech vaccine. ${ }^{2}$

\section{Symptoms}

The NICE guideline has been adapted from guidance produced by the UK Expert Haematology Panel in May. ${ }^{3}$ VITT should be suspected if a patient has any of the following symptoms five to 30 days after having a covid-19 vaccination:

- New onset of severe headache, which is getting worse and does not respond to simple painkillers;

- An unusual headache that seems worse when lying down or bending over or may be accompanied by blurred vision, nausea and vomiting, difficulty with speech, weakness, drowsiness, or seizures;

- New, unexplained pinprick bruising or bleeding; or

- Shortness of breath, chest pain, leg swelling, or persistent abdominal pain.

If a full blood count confirms thrombocytopenia or if a strong clinical suspicion of VITT remains, a coagulation screen should be carried out in secondary care, including a fibrinogen assay and D-dimer measurement. The condition can be confirmed using an enzyme linked immunosorbent assay (ELISA) to detect platelet factor 4 antibodies ( $\left.\mathrm{PF}_{4}\right)$.

The guideline recommends treatment options for people with VITT, including non-heparin anticoagulation drugs and surgical interventions to treat thrombosis. Patients would also initially receive intravenous immunoglobulin to treat the VITT immune response.

If blood tests indicate that someone is unlikely to have VITT, doctors should discuss the signs and symptoms of the condition with them and give advice on when and where to get further medical attention if the symptoms persist or worsen. Doctors should consider repeating the full blood count after two to three days if symptoms worsen.

The guideline will be continuously updated to incorporate new evidence when it becomes available. It has been published on the MAGICapp platform, a global system that allows rapid sharing of evidence between guideline developers.

1 National Institute for Health and Care Excellence. Covid-19 rapid guideline: vaccine-induced immune thrombocytopenia and thrombosis (VITT) NICE guideline [NG200]. 29 Jul 2021. https://www. nice.org.uk/guidance/NG200

2 Medicines and Healthcare Products Regulatory Agency. Research and analysis: Coronavirus vaccine-weekly summary of Yellow Card reporting. Updated 22 Jul 2021. https://www.gov.uk/government/publications/coronavirus-covid-19-vaccine-adverse-reactions/coronavirus-vaccine-summaryof-yellow-card-reporting

3 Wise J. Covid-19: Rare immune response may cause clots after AstraZeneca vaccine, say researchers. BMJ 2021;373:n954. doi: 10.1136/bmj.n954 pmid: 33846162

This article is made freely available for use in accordance with BMJ's website terms and conditions for the duration of the covid-19 pandemic or until otherwise determined by BMJ. You may use, download and print the article for any lawful, non-commercial purpose (including text and data mining) provided that all copyright notices and trade marks are retained. 